\title{
Biochemical Changes in Storage of Groundnut Seeds Under Different Locations, Storage Containers and Storage Conditions
}

\author{
M. Sujatha*, D.S. Uppar, N.K. Biradarpatil, U.V. Mummigatti and Nagaratna S. Olekar \\ Department of Seed Science and Technology, University of Agricultural Sciences, Dharwad- \\ 580005, India \\ *Corresponding author
}

\section{A B S T R A C T}

\begin{abstract}
Keywords
cold storage,

Vacuum pack,

Lipase enzyme,

Protease enzyme,

Groundnut

Article Info

Accepted:

04 June 2018

Available Online:

10 July 2018

The studies were carried out to evaluate the biochemical changes in the groundnut seeds stored in different locations, storage conditions and containers during 12 months of storage. Result from the study indicated that the Bagalkot produced seeds stored in cold storage conditions and packed in vacuum pack maintained higher seed quality parameters viz., higher oil content $(47.78 \%)$, protein content $(31.19 \%)$ and lower lipase $(0.53 \mu$ moles $\left.\mathrm{min}^{-1}\right)$ and protease activity $(0.262 \mathrm{U} / \mathrm{ml})$ after 12 months of storage period as compared to Dharwad and Kumta locations, ambient condition and other containers. Oil and protein content decreased while lipase and protease activity increased with the advancement of storage period. It concluded that cold storage condition and vacuum packing be used for storage of groundnut seeds in order to maintain seed viability for longer period.
\end{abstract}

\section{Introduction}

Groundnut (Arachis hypogaea L.) is a valuable food and oilseed crop. It is commonly called as the King of vegetable oilseeds crops or Poor man's nut. It is one of the poor storer, low seedling vigour is largely due to deterioration of seeds during storage. Storage deterioration is a continuous process that occurs from the moment the seed is mature until it is planted. Seeds of all crop species stored under unfavourable conditions receive internal lesions from impaction, moisture stress or heat stress which may or may not be appreciated externally. The rate of deteriorative changes largely depends on the storage condition and initial quality of the seeds when placed in storage. The rapid loss of seed viability is observed in summer harvested groundnut and about 50 per cent viability could be lost within four to five months of storage (Nautiyal and Ravindra, 1991). Seed deterioration continues at varying rate depending upon pre-harvest environmental conditions in the field, seed moisture content, storage condition, storage temperature etc.

\section{Materials and Methods}

Seed materials (GPBD-4) required for storage experiments were produced during summer 
season of 2015-16 and stored in their respective places (ambient condition) viz., Dharwad, Bagalkot and Kumata and in cold storage condition at Dharwad and packed in HDPE bag, Polylined gunny bag and Vacuum packing for 12 months. Seed materials were collected bimonthly from respective places to assess the seed quality parameters in laboratory of National Seed Project, University of Agricultural Sciences, Dharwad.

\section{Results and Discussion}

Groundnut is an oil rich crop. Lipid rich seeds tend to have limited longevity. Therefore, the hypothesis of seed aging based on lipid degradation could be considered quite important. However, the rate of decline in oil content observed in the present study being slower in the beginning and rapid between 6 to 12 months of storage.

The oil content decreased progressively from initial 48.77 to 46.68 per cent at $12^{\text {th }}$ month of storage. There was a non-significant effect of provenances on oil content until $4^{\text {th }}$ month of storage. Oil content was found to differ significantly between the provenances only after $4^{\text {th }}$ month of storage. Seeds produced at Bagalkot $\left(\mathrm{L}_{2}\right)$ and Dharwad $\left(\mathrm{L}_{1}\right)$ recorded significantly higher oil content (47.00 and $46.68 \%$ ) over Kumta $\left(\mathrm{L}_{3}\right)$ at the end of storage $(45.90 \%)$. But the lipase activity increased progressively from initial 0.50 to $0.71 \mu$ moles $\min ^{-1}$ at the end of $12^{\text {th }}$ month. After $6^{\text {th }}$ month of storage lipase activity differed significantly due to provenances. Among them, the seeds produced and stored at Bagalkot had significantly lower lipase activity $\left(0.62 \mu\right.$ moles $\left.\mathrm{min}^{-1}\right)$ compared to Dharwad (0.69 $\mu$ moles $\min ^{-1}$ ) and Kumta $\left(0.82 \mu\right.$ moles $\left.\mathrm{min}^{-1}\right)$ at the end of storage (Table 1). As the seeds produced and stored in Kumta are exposed to higher temperature and relative humidity, the enzymatic activity in the seeds were very high as compared to other locations. So because of higher activity of lipase enzyme the reduction in the oil content was more in Kumta

Oilseeds are rich sources of triacylglycerols and any increase in lipase activity is likely to accelerate the breakdown of triacylglycerols to glycerol and fatty acid which adversely affects the stored seeds. Significant difference for oil content was noticed among the containers after $4^{\text {th }}$ month of storage. Seeds stored under vacuum packed bag $\left(\mathrm{B}_{3}\right)$ recorded significantly higher oil content $(47.56 \%)$ as compared to that of poly lined gunny bag $\left(\mathrm{B}_{2}\right)$ and HDPE bag $\left(\mathrm{B}_{1}\right)$ which recorded oil content of 47.03 and 45.01 per cent respectively at the end of storage. Whereas, lipase activity among storage containers and storage conditions differed significantly after $6^{\text {th }}$ month of storage and it was less in vacuum packed seeds $\left(0.65 \mu\right.$ moles $\left.\mathrm{min}^{-1}\right)$, followed by polylined gunny bag and HDPE bag $(0.75$ $\mu$ moles $\min ^{-1}$ and $0.92 \mu$ moles $\min ^{-1}$ respectively) at $12^{\text {th }}$ month (Table 1 and Fig. $1)$.

Cold storage condition $\left(\mathrm{S}_{1}\right)$ recorded significantly higher oil content $(46.69 \%)$ as compared to that of ambient storage condition $\left(\mathrm{S}_{2}\right)$ at the end of storage $(46.37 \%)$. In contrast less lipase activity $(0.69 \mu$ moles $\min ^{-1}$ ) was recorded in cold storage condition, which was significantly different when compared to ambient storage condition $(0.73 \mu$ moles $\mathrm{min}^{-1}$ ) at the end of 12 months of storage (Table 1). Higher lipase enzyme activity was found in the seeds stored at higher temperature where the enzyme acted upon oil content to form simpler forms, so the oil content was found to be lower in seeds stored at ambient condition compare to cold storage condition.

Among interaction of locations, storage conditions and storage containers, the seeds produced in Bagalkot and stored in vaccum 
packing under cold storage condition recorded higher oil content $(47.78 \%)$ and lower lipase activity $\left(0.53 \mu\right.$ moles $\left.\mathrm{min}^{-1}\right)$, followed by seeds produced in Dharwad and stored in vaccum packing under cold storage condition (47.62 \% and $0.55 \mu$ moles $\min ^{-1}$ ) and the lowest oil content and higher lipase activity was recorded in seeds produced and stored in Kumta in HDPE bags under ambient condition $\left(44.39 \%\right.$ and $0.97 \mu$ moles $\min ^{-1}$ ) at the end of $12^{\text {th }}$ month of storage (Table 1$)$. The decrease rate in total lipid content is likely a consequence of fast metabolism by the seeds under conditions of high temperature and humidity. Changes in enzyme activities during aging process in groundnut were evident from increase in lipase activity in seeds stored under ambient condition. This could be another reason for the deterioration of seeds during storage since lipase is the principal enzymes involved in the degradation of lipids in seeds. The results in the present study are similar to that of Sultana (1994) and Mahantesh (2002) in groundnut.

Fig.1 Influence of provenances, storage containers and seed forms on oil content and lipase enzyme in summer groundnut stored under ambient condition

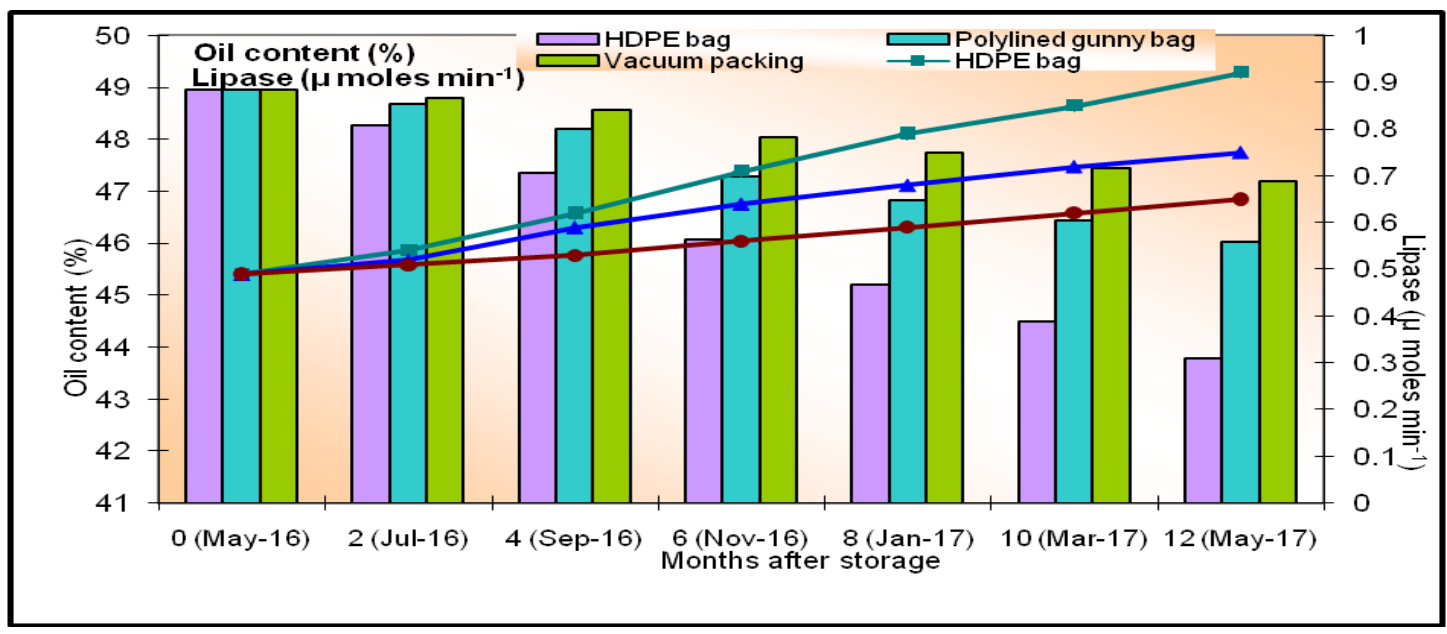

Fig.2 Influence of provenances, storage containers and seed forms on protein content and protease enzyme in summer groundnut stored under ambient condition

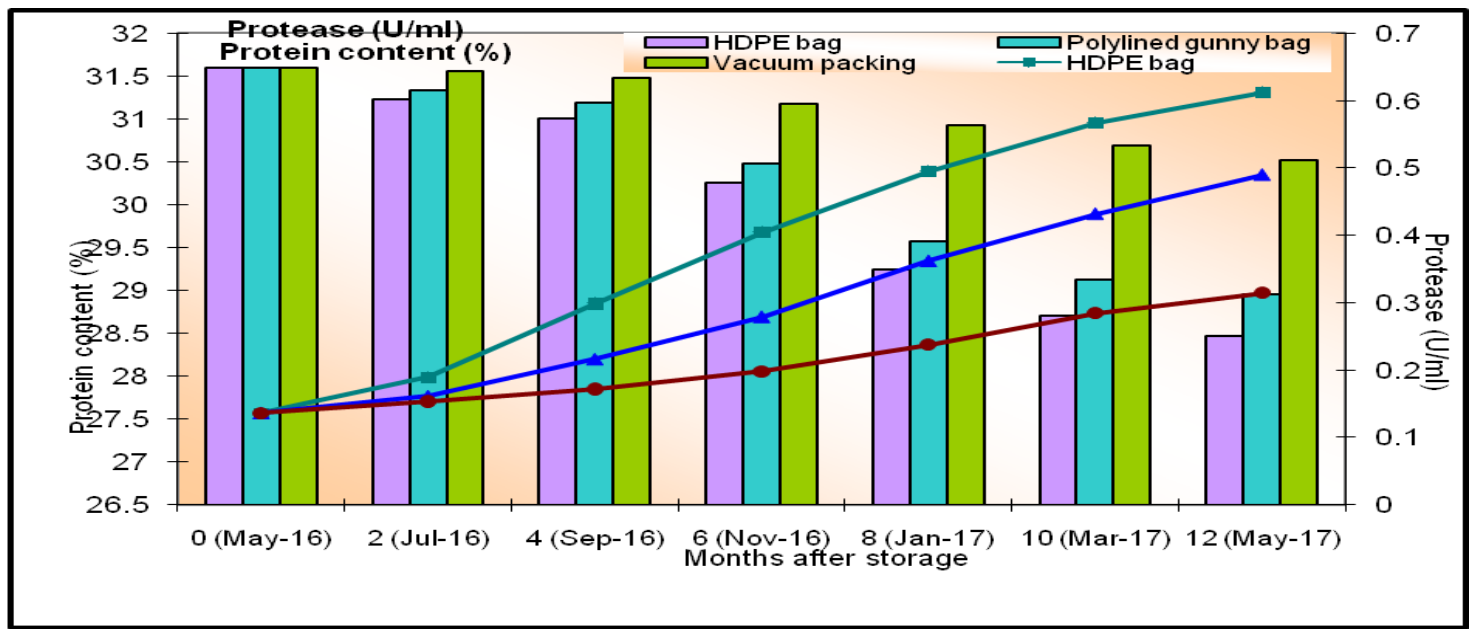


Table.1 Effect of provenance, storage conditions and storage containers on oil content (\%) and lipase $\left(\mu \operatorname{moles~min}^{-1}\right)$ in summer groundnut

\begin{tabular}{|c|c|c|c|c|c|c|c|c|c|}
\hline \multirow{2}{*}{\multicolumn{2}{|c|}{ Treatments }} & \multicolumn{4}{|c|}{ Oil content $\%$ (Months after storage) } & \multicolumn{4}{|c|}{ Lipase $\left(\mu\right.$ moles $\left.\min ^{-1}\right)$ (Months after storage) } \\
\hline & & \multirow{2}{*}{$\begin{array}{c}\text { Initial } \\
48.77\end{array}$} & \multirow{2}{*}{$\begin{array}{c}4^{\text {th }} \text { month } \\
48.14\end{array}$} & \multirow{2}{*}{$\begin{array}{c}8^{\text {th }} \text { month } \\
47.20\end{array}$} & \multirow{2}{*}{$\begin{array}{c}\text { 12 }^{\text {th }} \text { month } \\
46.68\end{array}$} & \multirow{2}{*}{$\begin{array}{c}\text { Initial } \\
0.50\end{array}$} & \multirow{2}{*}{$\begin{array}{c}4^{\text {th }} \text { month } \\
0.57\end{array}$} & \multirow{2}{*}{$\begin{array}{c}8^{\text {th }} \text { month } \\
0.64\end{array}$} & \multirow{2}{*}{$\begin{array}{c}\mathbf{1 2}^{\text {th }} \text { month } \\
0.69\end{array}$} \\
\hline Locations (L) & Dharwad $\left(\mathrm{L}_{1}\right)$ & & & & & & & & \\
\hline & Bagalkot $\left(\mathrm{L}_{2}\right)$ & 48.96 & 48.39 & 47.49 & 47.00 & 0.49 & 0.51 & 0.57 & 0.62 \\
\hline & Kumta $\left(\mathrm{L}_{3}\right)$ & 49.22 & 48.25 & 46.93 & 45.90 & 0.50 & 0.58 & 0.70 & 0.82 \\
\hline & Mean & 48.98 & 48.26 & 47.20 & 46.53 & 0.50 & 0.55 & 0.64 & 0.71 \\
\hline & S. Em. \pm & 0.21 & 0.22 & 0.16 & 0.23 & 0.009 & 0.012 & 0.008 & 0.007 \\
\hline & C. D. $(0.01)$ & NS & NS & 0.58 & 0.85 & NS & NS & 0.030 & 0.025 \\
\hline \multirow{5}{*}{$\begin{array}{l}\text { Storage conditions } \\
\text { (S) }\end{array}$} & Ambient $\left(\mathrm{S}_{1}\right)$ & 49.00 & 48.19 & 47.03 & 46.37 & 0.49 & 0.57 & 0.66 & 0.73 \\
\hline & $\operatorname{Cold}\left(\mathrm{S}_{2}\right)$ & 48.96 & 48.33 & 47.38 & 46.69 & 0.50 & 0.53 & 0.61 & 0.69 \\
\hline & Mean & 48.98 & 48.26 & 47.20 & 46.53 & 0.50 & 0.55 & 0.64 & 0.71 \\
\hline & S. Em. \pm & 0.18 & 0.18 & 0.13 & 0.19 & 0.007 & 0.010 & 0.007 & 0.005 \\
\hline & C. D. $(0.01)$ & NS & NS & 0.47 & 0.69 & NS & NS & 0.024 & 0.020 \\
\hline \multirow{6}{*}{$\begin{array}{l}\text { Bags for storage } \\
\text { (B) }\end{array}$} & HDPE bag $\left(\mathrm{B}_{1}\right)$ & 48.98 & 47.70 & 46.12 & 45.01 & 0.50 & 0.58 & 0.73 & 0.84 \\
\hline & $\begin{array}{l}\text { Polylined gunny bag } \\
\left(\mathrm{B}_{2}\right)\end{array}$ & 48.98 & 48.42 & 47.56 & 47.03 & 0.50 & 0.56 & 0.62 & 0.68 \\
\hline & Vacuum packing $\left(\mathrm{B}_{3}\right)$ & 48.98 & 48.66 & 47.93 & 47.56 & 0.50 & 0.52 & 0.56 & 0.61 \\
\hline & Mean & 48.98 & 48.26 & 47.20 & 46.53 & 0.50 & 0.55 & 0.64 & 0.71 \\
\hline & S. Em. \pm & 0.21 & 0.22 & 0.16 & 0.23 & 0.009 & 0.012 & 0.008 & 0.007 \\
\hline & C. D. $(0.01)$ & NS & NS & 0.58 & 0.85 & NS & NS & 0.030 & 0.025 \\
\hline \multirow[t]{9}{*}{ Interaction $(\mathrm{L} \times \mathrm{S})$} & $\mathrm{L}_{1} \mathrm{~S}_{1}$ & 48.77 & 48.06 & 46.99 & 46.46 & 0.50 & 0.60 & 0.68 & 0.73 \\
\hline & $\mathrm{L}_{1} \mathrm{~S}_{2}$ & 48.77 & 48.22 & 47.40 & 46.91 & 0.50 & 0.53 & 0.59 & 0.64 \\
\hline & $\mathrm{L}_{2} \mathrm{~S}_{1}$ & 49.01 & 48.36 & 47.28 & 46.78 & 0.48 & 0.52 & 0.58 & 0.63 \\
\hline & $\mathrm{L}_{2} \mathrm{~S}_{2}$ & 48.90 & 48.42 & 47.69 & 47.23 & 0.50 & 0.51 & 0.56 & 0.61 \\
\hline & $\mathrm{L}_{3} \mathrm{~S}_{1}$ & 49.22 & 48.14 & 46.81 & 45.88 & 0.50 & 0.58 & 0.71 & 0.83 \\
\hline & $\mathrm{L}_{3} \mathrm{~S}_{2}$ & 49.22 & 48.36 & 47.05 & 45.93 & 0.50 & 0.57 & 0.69 & 0.81 \\
\hline & Mean & 48.98 & 48.26 & 47.20 & 46.53 & 0.50 & 0.55 & 0.64 & 0.71 \\
\hline & S. Em. \pm & 0.30 & 0.31 & 0.22 & 0.32 & 0.012 & 0.017 & 0.011 & 0.009 \\
\hline & C. D. $(0.01)$ & NS & NS & 0.82 & 1.20 & NS & NS & 0.042 & 0.035 \\
\hline \multirow[t]{8}{*}{ Interaction $(\mathrm{L} \times \mathrm{B})$} & $\mathrm{L}_{1} \mathrm{~B}_{1}$ & 48.77 & 47.57 & 46.09 & 45.15 & 0.50 & 0.58 & 0.71 & 0.80 \\
\hline & $\mathrm{L}_{1} \mathrm{~B}_{2}$ & 48.77 & 48.36 & 47.68 & 47.35 & 0.50 & 0.61 & 0.67 & 0.70 \\
\hline & $\mathrm{L}_{1} \mathrm{~B}_{3}$ & 48.77 & 48.49 & 47.82 & 47.56 & 0.50 & 0.51 & 0.54 & 0.56 \\
\hline & $\mathrm{L}_{2} \mathrm{~B}_{1}$ & 48.96 & 47.86 & 46.44 & 45.56 & 0.49 & 0.55 & 0.67 & 0.76 \\
\hline & $\mathrm{L}_{2} \mathrm{~B}_{2}$ & 48.96 & 48.61 & 48.01 & 47.70 & 0.49 & 0.50 & 0.53 & 0.57 \\
\hline & $\mathrm{L}_{2} \mathrm{~B}_{3}$ & 48.96 & 48.71 & 48.01 & 47.74 & 0.49 & 0.49 & 0.51 & 0.53 \\
\hline & $\mathrm{L}_{3} \mathrm{~B}_{1}$ & 49.22 & 47.69 & 45.84 & 44.32 & 0.50 & 0.61 & 0.80 & 0.96 \\
\hline & $\mathrm{L}_{3} \mathrm{~B}_{2}$ & 49.22 & 48.30 & 46.98 & 46.03 & 0.50 & 0.56 & 0.66 & 0.77 \\
\hline
\end{tabular}


Int.J.Curr.Microbiol.App.Sci (2018) 7(7): 297-305

Table 1. Contd.....

\begin{tabular}{|c|c|c|c|c|c|c|c|c|c|}
\hline & $\mathrm{L}_{3} \mathrm{~B}_{3}$ & 49.22 & 48.77 & 47.97 & 47.37 & 0.50 & 0.55 & 0.64 & 0.73 \\
\hline & Mean & 48.98 & 48.26 & 47.20 & 46.53 & 0.50 & 0.55 & 0.64 & 0.71 \\
\hline & S. Em. \pm & 0.37 & 0.38 & 0.27 & 0.39 & 0.015 & 0.021 & 0.014 & 0.012 \\
\hline & C. D. $(0.01)$ & NS & NS & 1.00 & 1.47 & NS & NS & 0.052 & 0.043 \\
\hline \multirow[t]{9}{*}{ Interaction $(\mathrm{S} \times \mathrm{B})$} & $\mathrm{S}_{1} \mathrm{~B}_{1}$ & 49.00 & 47.55 & 45.80 & 44.73 & 0.49 & 0.59 & 0.75 & 0.86 \\
\hline & $\mathrm{S}_{1} \mathrm{~B}_{2}$ & 49.00 & 48.36 & 47.43 & 46.88 & 0.49 & 0.60 & 0.66 & 0.72 \\
\hline & $\mathrm{S}_{1} \mathrm{~B}_{3}$ & 49.00 & 48.64 & 47.85 & 47.50 & 0.49 & 0.52 & 0.57 & 0.62 \\
\hline & $\mathrm{S}_{2} \mathrm{~B}_{1}$ & 48.96 & 47.85 & 46.44 & 45.29 & 0.50 & 0.57 & 0.70 & 0.82 \\
\hline & $\mathrm{S}_{2} \mathrm{~B}_{2}$ & 48.96 & 48.48 & 47.69 & 47.17 & 0.50 & 0.52 & 0.58 & 0.64 \\
\hline & $\mathrm{S}_{2} \mathrm{~B}_{3}$ & 48.96 & 48.67 & 48.02 & 47.61 & 0.50 & 0.52 & 0.56 & 0.60 \\
\hline & Mean & 48.98 & 48.26 & 47.20 & 46.53 & 0.50 & 0.55 & 0.64 & 0.71 \\
\hline & S. Em. \pm & 0.37 & 0.38 & 0.27 & 0.39 & 0.012 & 0.017 & 0.011 & 0.009 \\
\hline & C. D. $(0.01)$ & NS & NS & 1.00 & 1.47 & NS & NS & 0.042 & 0.035 \\
\hline \multirow{21}{*}{$\begin{array}{l}\text { Interaction } \\
(\mathrm{L} \times \mathrm{S} \times \mathrm{B})\end{array}$} & $\mathrm{L}_{1} \mathrm{~S}_{1} \mathrm{~B}_{1}$ & 48.77 & 47.40 & 45.66 & 44.69 & 0.50 & 0.59 & 0.74 & 0.84 \\
\hline & $\mathrm{L}_{1} \mathrm{~S}_{1} \mathrm{~B}_{2}$ & 48.77 & 48.31 & 47.55 & 47.20 & 0.50 & 0.71 & 0.76 & 0.80 \\
\hline & $\mathrm{L}_{1} \mathrm{~S}_{1} \mathrm{~B}_{3}$ & 48.77 & 48.47 & 47.77 & 47.49 & 0.50 & 0.51 & 0.54 & 0.57 \\
\hline & $\mathrm{L}_{1} \mathrm{~S}_{2} \mathrm{~B}_{1}$ & 48.77 & 47.73 & 46.52 & 45.61 & 0.50 & 0.56 & 0.68 & 0.77 \\
\hline & $\mathrm{L}_{1} \mathrm{~S}_{2} \mathrm{~B}_{2}$ & 48.77 & 48.41 & 47.81 & 47.50 & 0.50 & 0.51 & 0.57 & 0.60 \\
\hline & $\mathrm{L}_{1} \mathrm{~S}_{2} \mathrm{~B}_{3}$ & 48.77 & 48.51 & 47.88 & 47.62 & 0.50 & 0.51 & 0.53 & 0.55 \\
\hline & $\mathrm{L}_{2} \mathrm{~S}_{1} \mathrm{~B}_{1}$ & 49.01 & 47.73 & 46.03 & 45.09 & 0.48 & 0.56 & 0.69 & 0.78 \\
\hline & $\mathrm{L}_{2} \mathrm{~S}_{1} \mathrm{~B}_{2}$ & 49.01 & 48.61 & 47.86 & 47.53 & 0.48 & 0.51 & 0.54 & 0.58 \\
\hline & $\mathrm{L}_{2} \mathrm{~S}_{1} \mathrm{~B}_{3}$ & 49.01 & 48.75 & 47.96 & 47.70 & 0.48 & 0.49 & 0.51 & 0.54 \\
\hline & $\mathrm{L}_{2} \mathrm{~S}_{2} \mathrm{~B}_{1}$ & 48.90 & 47.98 & 46.85 & 46.02 & 0.50 & 0.54 & 0.65 & 0.74 \\
\hline & $\mathrm{L}_{2} \mathrm{~S}_{2} \mathrm{~B}_{2}$ & 48.90 & 48.61 & 48.15 & 47.88 & 0.50 & 0.49 & 0.52 & 0.55 \\
\hline & $\mathrm{L}_{2} \mathrm{~S}_{2} \mathrm{~B}_{3}$ & 48.90 & 48.68 & 48.06 & 47.78 & 0.50 & 0.49 & 0.51 & 0.53 \\
\hline & $\mathrm{L}_{3} \mathrm{~S}_{1} \mathrm{~B}_{1}$ & 49.22 & 47.53 & 45.73 & 44.39 & 0.50 & 0.62 & 0.82 & 0.97 \\
\hline & $\mathrm{L}_{3} \mathrm{~S}_{1} \mathrm{~B}_{2}$ & 49.22 & 48.17 & 46.87 & 45.92 & 0.50 & 0.57 & 0.68 & 0.78 \\
\hline & $\mathrm{L}_{3} \mathrm{~S}_{1} \mathrm{~B}_{3}$ & 49.22 & 48.71 & 47.82 & 47.32 & 0.50 & 0.56 & 0.64 & 0.74 \\
\hline & $\mathrm{L}_{3} \mathrm{~S}_{2} \mathrm{~B}_{1}$ & 49.22 & 47.85 & 45.95 & 44.24 & 0.50 & 0.60 & 0.78 & 0.95 \\
\hline & $\mathrm{L}_{3} \mathrm{~S}_{2} \mathrm{~B}_{2}$ & 49.22 & 48.42 & 47.09 & 46.14 & 0.50 & 0.55 & 0.65 & 0.75 \\
\hline & $\mathrm{L}_{3} \mathrm{~S}_{2} \mathrm{~B}_{3}$ & 49.22 & 48.82 & 48.12 & 47.42 & 0.50 & 0.55 & 0.63 & 0.72 \\
\hline & Mean & 48.98 & 48.26 & 47.20 & 46.53 & 0.50 & 0.55 & 0.64 & 0.71 \\
\hline & S. Em. \pm & 0.53 & 0.54 & 0.38 & 0.56 & 0.021 & 0.030 & 0.020 & 0.016 \\
\hline & C. D. $(0.01)$ & NS & NS & 1.42 & 2.08 & NS & NS & 0.073 & 0.061 \\
\hline
\end{tabular}


Table.2 Effect of provenance, storage conditions and storage containers on protein content (\%) and protease (U/ml) in summer groundnut

\begin{tabular}{|c|c|c|c|c|c|c|c|c|c|}
\hline \multicolumn{2}{|c|}{ Treatments } & \multicolumn{4}{|c|}{ Protein content \% (Months after storage) } & \multicolumn{4}{|c|}{ Protease $(\mathrm{U} / \mathrm{ml})$ (Months after storage) } \\
\hline Locations (L) & Dharwad $\left(\mathrm{L}_{1}\right)$ & $\begin{array}{c}\text { Initial } \\
30.87\end{array}$ & $\begin{array}{c}4^{\text {th }} \text { month } \\
31.37\end{array}$ & $\begin{array}{c}8^{\text {th }} \text { month } \\
30.40\end{array}$ & $\begin{array}{c}12^{\text {th }} \text { month } \\
29.85\end{array}$ & $\begin{array}{c}\text { Initial } \\
0.134\end{array}$ & $4^{\text {th }}$ month & $8^{\text {th }}$ month & $\begin{array}{c}\text { 12 }^{\text {th }} \text { month } \\
0.389\end{array}$ \\
\hline & Bagalkot $\left(\mathrm{L}_{2}\right)$ & 31.79 & 31.53 & 30.62 & 30.14 & 0.128 & 0.186 & 0.298 & 0.366 \\
\hline & Kumta $\left(\mathrm{L}_{3}\right)$ & 31.88 & 30.96 & 29.59 & 29.14 & 0.147 & 0.221 & 0.320 & 0.415 \\
\hline & Mean & 31.51 & 31.29 & 30.20 & 29.71 & 0.136 & 0.203 & 0.308 & 0.390 \\
\hline & S. Em. \pm & 0.18 & 0.19 & 0.19 & 0.23 & 0.007 & 0.009 & 0.002 & 0.006 \\
\hline & C. D. $(0.01)$ & NS & NS & 0.72 & 0.84 & NS & NS & 0.009 & 0.022 \\
\hline \multirow{5}{*}{$\begin{array}{l}\text { Storage conditions } \\
\text { (S) }\end{array}$} & Ambient $\left(\mathrm{S}_{1}\right)$ & 31.44 & 31.27 & 30.04 & 29.51 & 0.136 & 0.210 & 0.326 & 0.419 \\
\hline & Cold $\left(S_{2}\right)$ & 31.59 & 31.31 & 30.36 & 29.91 & 0.136 & 0.196 & 0.290 & 0.361 \\
\hline & Mean & 31.51 & 31.29 & 30.20 & 29.71 & 0.136 & 0.203 & 0.308 & 0.390 \\
\hline & S. Em. \pm & 0.15 & 0.15 & 0.16 & 0.18 & 0.006 & 0.007 & 0.002 & 0.005 \\
\hline & C. D. $(0.01)$ & NS & NS & 0.59 & 0.69 & NS & NS & 0.007 & 0.018 \\
\hline \multirow{6}{*}{$\begin{array}{l}\text { Bags for storage } \\
\text { (B) }\end{array}$} & HDPE bag $\left(\mathrm{B}_{1}\right)$ & 31.51 & 31.06 & 29.58 & 28.94 & 0.136 & 0.258 & 0.413 & 0.520 \\
\hline & $\begin{array}{l}\text { Polylined gunny bag } \\
\left(\mathrm{B}_{2}\right)\end{array}$ & 31.51 & 31.27 & 29.93 & 29.43 & 0.136 & 0.189 & 0.307 & 0.392 \\
\hline & Vacuum packing $\left(\mathrm{B}_{3}\right)$ & 31.51 & 31.53 & 31.09 & 30.77 & 0.136 & 0.162 & 0.203 & 0.258 \\
\hline & Mean & 31.51 & 31.29 & 30.20 & 29.71 & 0.136 & 0.203 & 0.308 & 0.390 \\
\hline & S. Em. \pm & 0.18 & 0.19 & 0.19 & 0.23 & 0.007 & 0.009 & 0.002 & 0.006 \\
\hline & C. D. $(0.01)$ & 0.68 & 0.70 & 0.72 & 0.84 & NS & NS & 0.009 & 0.022 \\
\hline \multirow[t]{9}{*}{ Interaction $(\mathrm{L} \times \mathrm{S})$} & $\mathrm{L}_{1} \mathrm{~S}_{1}$ & 30.08 & 31.35 & 30.26 & 29.67 & 0.134 & 0.216 & 0.326 & 0.422 \\
\hline & $\mathrm{L}_{1} \mathrm{~S}_{2}$ & 31.65 & 31.40 & 30.53 & 30.03 & 0.134 & 0.190 & 0.286 & 0.356 \\
\hline & $\mathrm{L}_{2} \mathrm{~S}_{1}$ & 31.79 & 31.52 & 30.50 & 30.00 & 0.128 & 0.187 & 0.297 & 0.371 \\
\hline & $\mathrm{L}_{2} \mathrm{~S}_{2}$ & 31.79 & 31.54 & 30.73 & 30.29 & 0.128 & 0.185 & 0.298 & 0.361 \\
\hline & $\mathrm{L}_{3} \mathrm{~S}_{1}$ & 32.44 & 30.92 & 29.36 & 28.87 & 0.147 & 0.227 & 0.356 & 0.466 \\
\hline & $\mathrm{L}_{3} \mathrm{~S}_{2}$ & 31.32 & 30.99 & 29.82 & 29.40 & 0.147 & 0.214 & 0.284 & 0.364 \\
\hline & Mean & 31.51 & 31.29 & 30.20 & 29.71 & 0.136 & 0.203 & 0.308 & 0.390 \\
\hline & S. Em. \pm & 0.26 & 0.27 & 0.27 & 0.32 & 0.010 & 0.013 & 0.003 & 0.008 \\
\hline & C. D. $(0.01)$ & 0.96 & 0.99 & 1.02 & 1.19 & NS & NS & 0.013 & 0.031 \\
\hline \multirow[t]{8}{*}{ Interaction $(\mathrm{L} \times \mathrm{B})$} & $\mathrm{L}_{1} \mathrm{~B}_{1}$ & 30.87 & 31.17 & 29.83 & 29.10 & 0.134 & 0.251 & 0.401 & 0.516 \\
\hline & $\mathrm{L}_{1} \mathrm{~B}_{2}$ & 30.87 & 31.35 & 30.15 & 29.57 & 0.134 & 0.195 & 0.305 & 0.390 \\
\hline & $\mathrm{L}_{1} \mathrm{~B}_{3}$ & 30.87 & 31.60 & 31.20 & 30.89 & 0.134 & 0.161 & 0.211 & 0.261 \\
\hline & $\mathrm{L}_{2} \mathrm{~B}_{1}$ & 31.79 & 31.31 & 30.08 & 29.41 & 0.128 & 0.243 & 0.393 & 0.488 \\
\hline & $\mathrm{L}_{2} \mathrm{~B}_{2}$ & 31.79 & 31.54 & 30.41 & 29.90 & 0.128 & 0.166 & 0.296 & 0.356 \\
\hline & $\mathrm{L}_{2} \mathrm{~B}_{3}$ & 31.79 & 31.74 & 31.37 & 31.12 & 0.128 & 0.149 & 0.204 & 0.254 \\
\hline & $\mathrm{L}_{3} \mathrm{~B}_{1}$ & 31.88 & 30.70 & 28.84 & 28.31 & 0.147 & 0.280 & 0.445 & 0.555 \\
\hline & $\mathrm{L}_{3} \mathrm{~B}_{2}$ & 31.88 & 30.91 & 29.24 & 28.82 & 0.147 & 0.207 & 0.320 & 0.430 \\
\hline
\end{tabular}


Int.J.Curr.Microbiol.App.Sci (2018) 7(7): 297-305

Table 1. Contd.....

\begin{tabular}{|c|c|c|c|c|c|c|c|c|c|}
\hline & $\mathrm{L}_{3} \mathrm{~B}_{3}$ & 31.88 & 31.26 & 30.70 & 30.29 & 0.147 & 0.175 & 0.195 & 0.260 \\
\hline & Mean & 31.51 & 31.29 & 30.20 & 29.71 & 0.136 & 0.203 & 0.308 & 0.390 \\
\hline & S. Em. \pm & 0.32 & 0.32 & 0.33 & 0.39 & 0.012 & 0.016 & 0.004 & 0.010 \\
\hline & C. D. $(0.01)$ & 1.18 & 1.21 & 1.25 & 1.45 & NS & NS & 0.016 & 0.038 \\
\hline \multirow[t]{9}{*}{ Interaction $(\mathrm{S} \times \mathrm{B})$} & $\mathrm{S}_{1} \mathrm{~B}_{1}$ & 31.44 & 31.03 & 29.38 & 28.68 & 0.136 & 0.268 & 0.438 & 0.561 \\
\hline & $\mathrm{S}_{1} \mathrm{~B}_{2}$ & 31.44 & 31.24 & 29.71 & 29.17 & 0.136 & 0.197 & 0.333 & 0.433 \\
\hline & $\mathrm{S}_{1} \mathrm{~B}_{3}$ & 31.44 & 31.52 & 31.04 & 30.70 & 0.136 & 0.165 & 0.208 & 0.265 \\
\hline & $\mathrm{S}_{2} \mathrm{~B}_{1}$ & 31.59 & 31.08 & 29.79 & 29.20 & 0.136 & 0.248 & 0.388 & 0.478 \\
\hline & $\mathrm{S}_{2} \mathrm{~B}_{2}$ & 31.59 & 31.29 & 30.15 & 29.69 & 0.136 & 0.181 & 0.281 & 0.351 \\
\hline & $\mathrm{S}_{2} \mathrm{~B}_{3}$ & 31.59 & 31.55 & 31.14 & 30.84 & 0.136 & 0.159 & 0.199 & 0.252 \\
\hline & Mean & 31.51 & 31.29 & 30.20 & 29.71 & 0.136 & 0.203 & 0.308 & 0.390 \\
\hline & S. Em. \pm & 0.26 & 0.27 & 0.27 & 0.32 & 0.010 & 0.013 & 0.003 & 0.008 \\
\hline & C. D. $(0.01)$ & 0.96 & 0.99 & 1.02 & 1.19 & NS & NS & 0.013 & 0.031 \\
\hline \multirow{21}{*}{$\begin{array}{c}\text { Interaction } \\
(\mathrm{L} \times \mathrm{S} \times \mathrm{B})\end{array}$} & $\mathrm{L}_{1} \mathrm{~S}_{1} \mathrm{~B}_{1}$ & 30.08 & 31.13 & 29.67 & 28.87 & 0.134 & 0.270 & 0.430 & 0.570 \\
\hline & $\mathrm{L}_{1} \mathrm{~S}_{1} \mathrm{~B}_{2}$ & 30.08 & 31.34 & 29.99 & 29.36 & 0.134 & 0.209 & 0.329 & 0.429 \\
\hline & $\mathrm{L}_{1} \mathrm{~S}_{1} \mathrm{~B}_{3}$ & 30.08 & 31.58 & 31.13 & 30.80 & 0.134 & 0.167 & 0.217 & 0.267 \\
\hline & $\mathrm{L}_{1} \mathrm{~S}_{2} \mathrm{~B}_{1}$ & 31.65 & 31.21 & 30.00 & 29.33 & 0.134 & 0.232 & 0.372 & 0.462 \\
\hline & $\mathrm{L}_{1} \mathrm{~S}_{2} \mathrm{~B}_{2}$ & 31.65 & 31.36 & 30.31 & 29.79 & 0.134 & 0.181 & 0.281 & 0.351 \\
\hline & $\mathrm{L}_{1} \mathrm{~S}_{2} \mathrm{~B}_{3}$ & 31.65 & 31.61 & 31.28 & 30.98 & 0.134 & 0.155 & 0.205 & 0.255 \\
\hline & $\mathrm{L}_{2} \mathrm{~S}_{1} \mathrm{~B}_{1}$ & 31.79 & 31.31 & 29.95 & 29.24 & 0.128 & 0.248 & 0.408 & 0.498 \\
\hline & $\mathrm{L}_{2} \mathrm{~S}_{1} \mathrm{~B}_{2}$ & 31.79 & 31.53 & 30.25 & 29.70 & 0.128 & 0.168 & 0.288 & 0.368 \\
\hline & $\mathrm{L}_{2} \mathrm{~S}_{1} \mathrm{~B}_{3}$ & 31.79 & 31.72 & 31.31 & 31.06 & 0.128 & 0.145 & 0.195 & 0.245 \\
\hline & $\mathrm{L}_{2} \mathrm{~S}_{2} \mathrm{~B}_{1}$ & 31.79 & 31.30 & 30.20 & 29.59 & 0.128 & 0.238 & 0.378 & 0.478 \\
\hline & $\mathrm{L}_{2} \mathrm{~S}_{2} \mathrm{~B}_{2}$ & 31.79 & 31.56 & 30.56 & 30.10 & 0.128 & 0.163 & 0.303 & 0.343 \\
\hline & $\mathrm{L}_{2} \mathrm{~S}_{2} \mathrm{~B}_{3}$ & 31.79 & 31.75 & 31.43 & 31.19 & 0.128 & 0.152 & 0.212 & 0.262 \\
\hline & $\mathrm{L}_{3} \mathrm{~S}_{1} \mathrm{~B}_{1}$ & 32.44 & 30.66 & 28.51 & 27.93 & 0.147 & 0.285 & 0.475 & 0.615 \\
\hline & $\mathrm{L}_{3} \mathrm{~S}_{1} \mathrm{~B}_{2}$ & 32.44 & 30.86 & 28.90 & 28.45 & 0.147 & 0.215 & 0.380 & 0.500 \\
\hline & $\mathrm{L}_{3} \mathrm{~S}_{1} \mathrm{~B}_{3}$ & 32.44 & 31.24 & 30.66 & 30.23 & 0.147 & 0.182 & 0.212 & 0.282 \\
\hline & $\mathrm{L}_{3} \mathrm{~S}_{2} \mathrm{~B}_{1}$ & 31.32 & 30.74 & 29.16 & 28.69 & 0.147 & 0.275 & 0.415 & 0.495 \\
\hline & $\mathrm{L}_{3} \mathrm{~S}_{2} \mathrm{~B}_{2}$ & 31.32 & 30.95 & 29.58 & 29.19 & 0.147 & 0.200 & 0.260 & 0.360 \\
\hline & $\mathrm{L}_{3} \mathrm{~S}_{2} \mathrm{~B}_{3}$ & 31.32 & 31.28 & 30.73 & 30.34 & 0.147 & 0.169 & 0.179 & 0.239 \\
\hline & Mean & 31.51 & 31.29 & 30.20 & 29.71 & 0.136 & 0.203 & 0.308 & 0.390 \\
\hline & S. Em. \pm & 0.45 & 0.46 & 0.47 & 0.55 & 0.017 & 0.022 & 0.006 & 0.014 \\
\hline & C. D. (0.01) & 1.67 & 1.71 & 1.76 & 2.06 & NS & NS & 0.022 & 0.054 \\
\hline
\end{tabular}


The protein content reduced progressively from initial $31.51 \mathrm{~cm}$ to 29.71 per cent at $12^{\text {th }}$ month of storage. After $4^{\text {th }}$ month of storage protein content differed significantly due to provenances. Among them, the seeds produced and stored at Bagalkot had significantly higher protein content $(30.14 \%)$ compared to Dharwad $(29.85 \%)$ and Kumta $(29.14 \%)$ at the end of storage. As the protease enzyme activity increased with storage, the protein content decreased. Seeds produced at Bagalkot $\left(\mathrm{L}_{2}\right)$ recorded significantly lower protease activity (0.366 U/ml) over Dharwad and Kumta (0.389 and $0.415 \mathrm{U} / \mathrm{ml}$ respectively) at the end of storage (Table 2).

Significant differences were noticed for protein content among storage containers after $4^{\text {th }}$ month of storage and it was more in vacuum packed seeds $(30.77 \%)$, followed by polylined gunny bag and HDPE bag (29.43 and $28.94 \%$ respectively) at $12^{\text {th }}$ month of storage. But protease activity was significantly less in vaccum packed bag $(0.258 \mathrm{U} / \mathrm{ml})$ as compared to polylines gunny bag $(0.392 \mathrm{U} / \mathrm{ml})$ and HDPE bag $(0.520$ $\mathrm{U} / \mathrm{ml}$ ) at the end of storage (Table 2 and Fig. 2).

Cold storage recorded significantly higher protein content and lower protease activity ( $29.91 \%$ and $0.361 \mathrm{U} / \mathrm{ml}$ ) when compared to ambient storage $(29.51 \%$ and $0.419 \mathrm{U} / \mathrm{ml})$ at the end of 12 months of storage (Table 2). Increase in lipase and protease activity during storage may be due to decline in oil and protein content. But rate of decline was less in cold storage. Similar results were observed with Naik (2013) in rice, Chaitanya et al., (2000) in Shorea robusta.

Among interaction of locations, storage conditions and storage containers, the seeds produced in Bagalkot and stored in vaccum packing under cold storage condition recorded higher protein content $(31.19 \%)$ and lower protease activity $(0.262 \mathrm{U} / \mathrm{ml})$ followed by seeds produced in Dharwad and stored in vaccum packing under cold storage condition $(30.98 \%$ and $0.255 \mathrm{U} / \mathrm{ml})$ and the lowest protein content and higher protease activity was recorded in seeds produced and stored in Kumta in HDPE bags under ambient condition $(28.51 \%$ and $0.615 \mathrm{U} / \mathrm{ml})$ at the end of $12^{\text {th }}$ month of storage (Table 2).

Gradual decline in total protein content due to corresponding increase in protease activity preceded loss of viability. Enhanced protease activity results in seeds may account for concomitant loss of total protein content during storage. A similar trend in increase of lipase and protease activity under ambient condition has been reported by Sultana (1994) in groundnut and Naik (2013) in rice.

There was increase in the activity of these enzymes with advancement in storage period. Lipase and protease enzymes were produced abundantly during storage which breaks down the lipid into free fatty acids and proteins into amino acids. Similar increase in these parameters during storage of seeds was reported by Meena (2014) and Begum (2014) in groundnut. Different longevity of seed storage as well as storage conditions exerts significant influence on oil content. The rate of reduction in oil and protein content was less in cold storage due to lesser lipase and protease activity and respiration rate at low temperature.

\section{References}

Begum, A. J. M., Balamurugan, P., Vanagamudi, K., Prabakar and Ramakrishnan, R., 2014. Enzyme changes during seed storage in groundnut (Arachis hypogaea L.). J. Appl. Natural. Sci. 6 (2): 748 - 750.

Chaitanya, K. S., Keshavkant, S. and 
Naithani, S. C., 2000. Changes in total protein and protease activity in dehydrating recalcitrant sal (Shorea robusta) seeds. Silva Fennica. 34(1): 71-77.

Mahantesh, M. B., 2002. Studies on seed quality and field performance of groundnut seeds stored under coastal conditions of Karnataka. M.Sc. (Agri.) Thesis, University of Agricultural Sciences, Bangalore.

Meena, M. K., 2014. Influence of vacuum packaging on seed physiology and quality in different field crops. Ph. D. Thesis, Univ. Agric. Sci., Dharwad, Karnataka (India).

Naik, S. D., 2013. Studies on physiological and biochemical changes during long term storage of paddy and rice under different packaging materials. Ph. D. Thesis, Univ. Agric. Sci., Dharwad, Karnataka (India).

Nautiyal, P. C., Ravindra, V., Vasantha, S. and Joshi. Y. C. 1991. Moisture stress and subsequent seed viability physiological and biochemical basis for viability differences in Spanish groundnut in response to soil moisture stress. Oleagineux. 46: 153-158.

Sultana, A., 1994. Seed aging and deterioration during storage for germplasm conservation in groundnut (Arachis hypogea L.) and its wild relatives. Ph. D. Thesis, Indian Institute of Technology, Karagpur, India.

\section{How to cite this article:}

Sujatha, M., D.S. Uppar, N.K. Biradarpatil, U.V. Mummigatti and Nagaratna S. Olekar. 2018. Biochemical Changes in Storage of Groundnut Seeds Under Different Locations, Storage Containers and Storage Conditions. Int.J.Curr.Microbiol.App.Sci. 7(07): 297-305. doi: https://doi.org/10.20546/ijcmas.2018.707.036 